\title{
ON THE TOXIN PRODUCTION OF THE DIPHTHERIA BACILLUS.
}

\author{
By ALFRED MaCCONKEY. \\ (Lister Institute, Elstree, Herts.) \\ (With 4 Charts.)
}

\begin{abstract}
EVERYONE who has been occupied with the weekly production of diphtheria toxin in quantity has been struck with the great variations which may and usually do occur in the strength of the toxin from week to week. It is indeed extraordinary as Madsen remarks (1908, p. 83) that a bacillus should for a long time give good and fairly constant results when grown in one laboratory and yet fail to do so when grown in another laboratory in a medium prepared with the greatest care according to the formula used in the first laboratory. That it is not merely a question of medium is shown by the following experiment. A batch of bouillon was made up and inoculated in the usual way. Then half the flasks were placed in one of the two hot rooms we have here, and the other half in the other hot room. After nine days' incubation the cultures were treated as usual. Both lots were tested on the same day and it was found that while the M.L.D. of the one half was $\frac{1}{150}$ c.cm., that of the other was $\frac{1}{50}$ c.cm. Many and varied have been the procedures suggested for growing the diphtheria bacillus so as to obtain a highly potent filtrate and most of them have been tried in this laboratory but none can be depended upon to give a constant result. When discussing this point Dean (1907, pp. 467-468) gives the M.L.D. of a large number of toxins prepared under his direction, but they are not given in order as they were prepared and so one cannot get an idea as to how one batch differed from the next. I have not come across any such series in the literature of this subject and so it seemed that it would be of interest to put on record a two years' series of results in the order in which they were obtained-more especially as a study of this series gave more food for thought than was anticipated.

The bouillon used was prepared according to the method recommended by Dean (loc. cit. p. 466) and the details are given here for convenience sake.
\end{abstract}


Take $1 \mathrm{lb}$. of lean beef (silverside ${ }^{1}$ ) freed from fat, mince it and add 1 litre of tap water.

Boil 1-2 hours and filter while hot.

To filtrate add $2 \%$ Witte's peptone and $0.5 \% \mathrm{NaCl}$ and steam 1 hour.

Neutralize with $\mathrm{Na}_{2} \mathrm{GO}_{3}$ solution using litmus paper as indicator and then add 7 c.c. $\frac{n}{1} \mathrm{NaOH}$ solution."

Steam 1 hour and filter cold.

Distribute into flasks².

Sterilize in the autoclave (steam for 20 mins.-raise the temperature to $134^{\circ} \mathrm{C}$. and then turn the gas off).

Each flask is inoculated with a small piece of the pellicle-growth from a flask which was seeded the previous week. The flasks are incubated at $36.5^{\circ} \mathrm{C}$. On the third day a flask is taken out of the incubator and kept in the ice chest until required to inoculate the next batch at the end of the week.

On the ninth day the cultures are filtered through paper ( $\mathrm{S}$ and $\mathrm{S}$ 597 ) and kept in Winchester quart glass-stoppered bottles, the surface of the toxin being covered with a layer of toluol. The bottles are placed in a cool dark cellar and the broth is shaken up each day for several days. At the end of a week, as all bacilli are dead, it is considered fit for testing, which usually takes place within a few days.

Chart No. 1 gives in the form of a curve the results obtained from week to week from September 1910 till July 1912. The ordinates represent the number of M.L.D.s per cubic centimetre and the abscissae the weeks.

As it was possible that toxin production might occur more quickly or more slowly during the summer I have tested the toxicity of cultures some of which had been grown for 3 and some for 14 days. In neither case was there any increase in strength over the 9 -day growth.

Considered simply from the point of view of weekly variations there is nothing to which one need call attention as the curve only depicts what has been an universal experience and one for which no explanation is forthcoming.

But if the curve be studied as a whole it is manifest that there is a rise and fall in the size of the minimal lethal dose during the summer

1 The silverside of beef is almost invariably used but it is not the only part which gives good results. It does not seem to matter whether the meat is used quite fresh (i.e. while still warm) or two days or several days old.

2 This broth is made up in batches of $10,20,30$ or 40 litres and kept in bulk. When required it is distributed in quantities of about 300-350 c.cm. into 1 litre Erlenmeyer flasks, which are sterilized in the autoclave and inoculated as soon as the bouillon is cool enough. 
and winter respectively and this rise and fall is not gradual. At the end of summer there is a rapid increase in the production of toxin and then the strength of the toxin remains high until about the end of March when a drop occurs and the toxin production remains low until the autumn.

It might be said that this seasonal variation was entirely due to changes in the resistance of the guinea-pig to the effects of diphtheria toxin-changes such as have been alluded to by Madsen (loc. cit. p. 90) and by Südmersen and Glenny (1909). While it is impossible to deny that this varying resistance may have some influence on the result still I do not think it can be accepted as the sole cause. From the

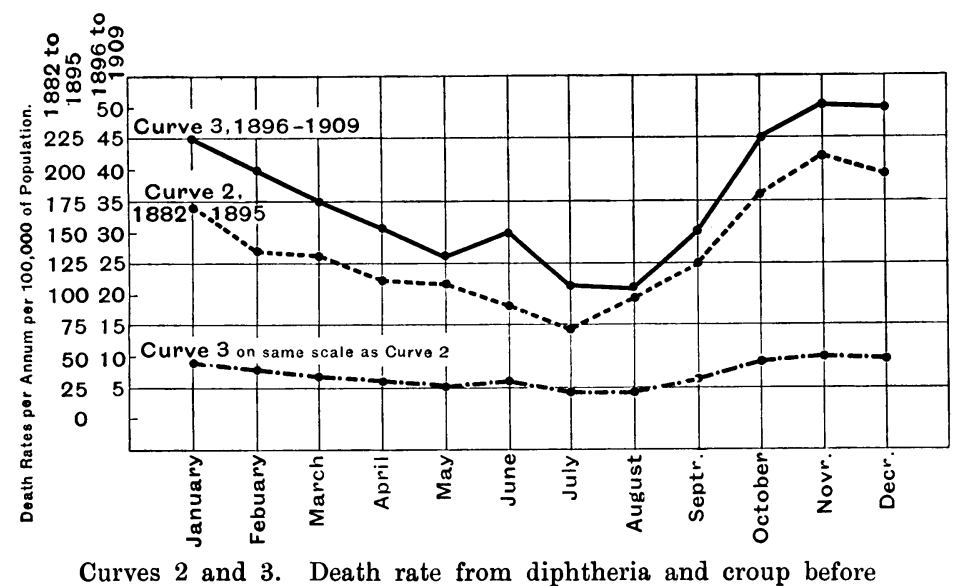

Curves 2 and 3. Death rate from diphtheria and croup before (2) and after (3) the introduction of antitoxin.

data which we have at our disposal we cannot come to any definite conclusion and therefore we may leave this point for discussion at a future date.

What I more particularly desire to call attention to is the similarity between the curve of toxin-production and certain other curves showing the incidence of diphtheria in the human subject.

The first two curves, Nos. 2 and 3, are from a leaflet issued by one of the American Health Authorities. They show the death-rate from diphtheria and croup during two 14-year periods-one before and the other after the introduction of antitoxin. In the leaflet both curves are of course drawn to the same scale but I have altered the scale of the second curve in one particular simply so as to make the fact that there is a rise and fall more easily appreciated. We see that though a large drop in the death-rate followed the use of antitoxin still a rise and fall occurred at the same seasons. 
Bearing in mind that these are the averages of 14 years and that in consequence abrupt changes must be to a considerable extent eliminated we cannot fail to be struck by the similarity between these two curves and the "toxin-curve"-especially in the rapid rise between July and November.

Curve 4 shows the number of cases of diphtheria notified week by week in the county of London and Curve 5 gives the number of cases of diphtheria admitted each week into the Metropolitan Asylums Board hospitals.

Now Südmersen and Glenny (p. 400) state that the toxin they studied apparently showed an increase in toxicity in one year compared with the previous year.

On directing our attention to this point we find that Curves 4 and 5 show that the number of cases of diphtheria notified or admitted into hospital was greater in the winter of 1911-12 than in that of 1910-11, and curve 1 shows that the strength of the various batches of diphtheria toxin reached and remained at a higher average level during 1911-12 than during 1910-11. We have then the curves of "toxin-production" and of "incidence in the human subject" agreeing in showing an annual as well as a seasonal variation. In fact the curves resemble each other in a remarkable and unexpected degree.

Naturally, we search for an explanation of this coincidence and we cannot find it as there are too many unknown factors in the problem. For instance, if we put these variations down to changes in the resistance of the animal organism, then we must admit that the susceptibility of all the people in the county of London has varied in much the same manner as that of our stock guinea-pigs. And on the other hand, if we consider them to be due to changes in the toxigenetic power of the bacillus, then we must allow that the offensive power of all the different strains of diphtheria bacilli in the county waxed and waned coincidently with that of our laboratory strain. Neither of these explanations is adequate and so we must leave the problem unsolved and be content with having drawn attention to the subject.

\section{REFERENCES.}

Dean, G. (1907). The Types of Immunity. The Bacteriology of Diphtheria, 449-573. Cambridge University Press.

Madsen, TH. (1908). "Diphtheria Toxin." Handbuch der Technik und Methodik der Immunitätsforschung, I. 71-102. Kraus und Levaditi.

SüDMersen, H. J. and Glenny, A. T. (1909). Variation in susceptibility of guineapigs to diphtheria toxin. Journ. of Hygiene, Ix. 399-408. 


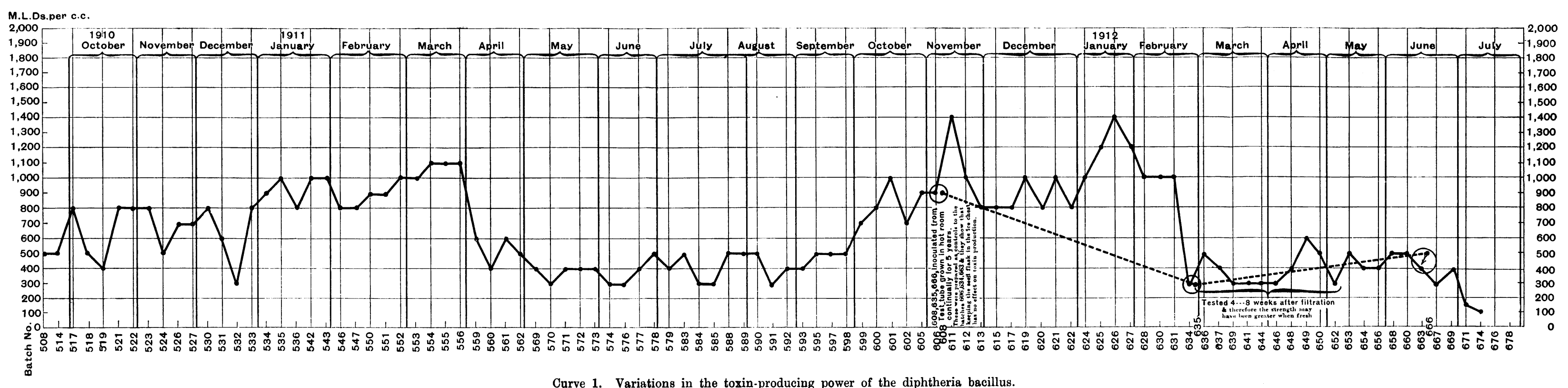

Curve 1. Variations in the toxin-producing power of the diphtheria bacillus.

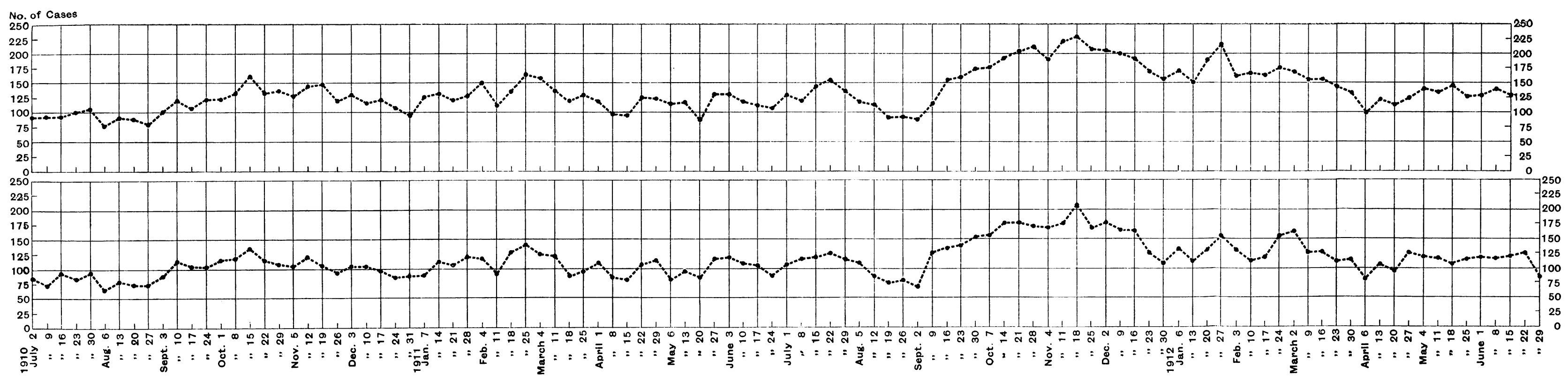

Curve 4. Cases of diphtheria notified each week in the County of London. 